\title{
Case Report \\ Superior Mesenteric Artery Dissection after Extracorporeal Shockwave Lithotripsy
}

\author{
Christos Bakoyiannis, ${ }^{1}$ Ioannis Anastasiou, ${ }^{2}$ \\ Andreas Koutsoumpelis, ${ }^{1}$ Evangelos Fragiadis, ${ }^{2}$ Eleni Felesaki, ${ }^{3}$ \\ Marina Kafeza, ${ }^{1}$ Sotirios Georgopoulos, ${ }^{1}$ and Christos Tsigris ${ }^{1}$ \\ ${ }^{1}$ Vascular Unit, First Department of Surgery, Laiko General Hospital, University of Athens, Athens, Greece \\ ${ }^{2}$ Department of Urology, Laiko General Hospital, University of Athens, Athens, Greece \\ ${ }^{3}$ Department of Radiology, Laiko General Hospital, Athens, Greece
}

Correspondence should be addressed to Andreas Koutsoumpelis, andk_79@yahoo.gr

Received 28 October 2012; Accepted 19 November 2012

Academic Editors: Y.-J. Chen and E. Minar

Copyright (C) 2012 Christos Bakoyiannis et al. This is an open access article distributed under the Creative Commons Attribution License, which permits unrestricted use, distribution, and reproduction in any medium, provided the original work is properly cited.

\begin{abstract}
The use of shockwave lithotripsy is currently the mainstay of treatment in renal calculosis. Several complications including vessel injuries have been implied to extracorporeal shockwave lithotripsy. We report an isolated dissection of the superior mesenteric artery in a 60-year-old male presenting with abdominal pain which occurred three days after extracorporeal shockwave lithotripsy. The patient was treated conservatively and the abdominal pain subsided 24 hours later. The patient's history, the course of his disease, and the timing may suggest a correlation between the dissection and the ESWL.
\end{abstract}

\section{Introduction}

The extracorporeal shockwave lithotripsy (ESWL) has become the gold standard in the treatment of renal calculosis especially in the upper lobe. As all therapeutic modalities, ESWL may present with several complications including arterial rupture or dissection regardless of the presence of aortic aneurysm [1-7]. The isolated dissection of the superior mesenteric artery, though, is very rare. We report a case of a 60-year-old male who developed isolated superior mesenteric artery dissection 5 days after ESWL.

\section{Case Report}

A 60-year-old male, ex-smoker, presented to the emergency department complaining of right colicky pain and highgrade fever 24 hours after being subjected to ESWL. Patient underwent an abdominal ultrasound revealing urolithiasis on the right side along with minimal urine stasis in the right renal pelvis. He was subjected to prophylactic antibiotic coverage and pigtail placement under fluoroscopic guidance with subsequent remission of fever and pain. Forty-eight hours later he complained of acute onset of sharp epigastric pain reflecting to the lumbar region. His physical examination revealed tenderness over the epigastrium with no abdominal palpable mass. The arterial pressure was mildly elevated $(140-90 \mathrm{mmHg})$ and his pulses were $60 \mathrm{bpm}$. His lab workout revealed a blood count and a basic metabolic panel within normal limits, with the exception of WBC and CRP which were moderately increased. An abdominal duplex ultrasound was performed revealing a flap in the superior mesenteric artery. The CT angiogram of thoracic and abdominal aorta demonstrated an isolated superior mesenteric artery dissection, starting from its orifice, without SMA branch involvement (Figure 1). Both true and false lumens were patent (Figure 2). The diameters of the infrarenal aorta and superior mesenteric artery were normal. Additionally, the aorta and the iliac arteries were found abundant of atheromatous plaques.

The patient was transferred to our vascular department. We decided to start a medical treatment consisting of food and liquid restriction, close surveillance, intravenous administration of beta-blockers, and antithrombotic therapy 


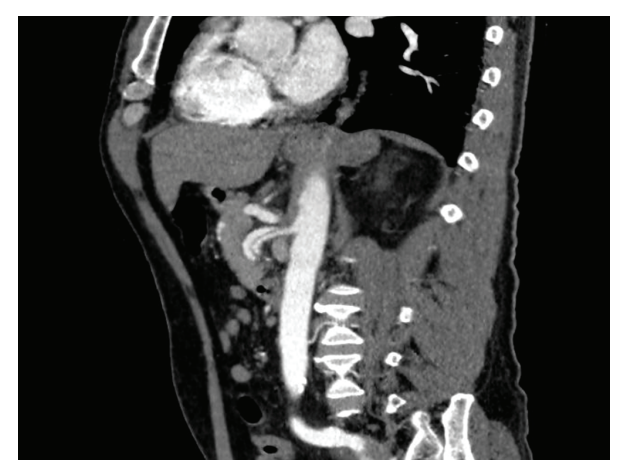

FIGURE 1: Computed tomography angiogram (sagittal view) depicting the dissection of the superior mesenteric artery extending from the orifice without involving any branch vessel.

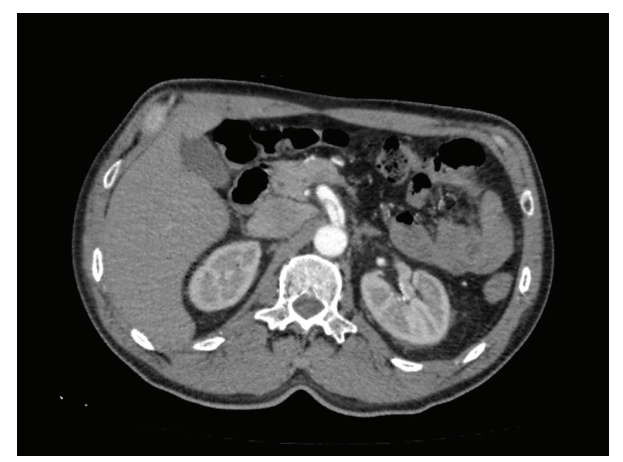

FIGURE 2: Computed tomography angiogram (axial view) revealing the double-barrel sign of the dissected superior mesenteric artery.

with unfractionated heparin. After 10 hours the patient's symptoms had diminished and subsided 24 hours later. Liquid restriction was halted on the third day and food restriction on the fourth day of admission to our department. No signs of bowel ischemia were reported during the patient's stay in our department. On the seventh day of admission, the patient underwent a CT angiography revealing no change in the arterial dissection. The patient was discharged ten days after admission and was advised to receive warfarin orally and to undergo a follow-up CT angiography one month later.

\section{Discussion}

The dissection of the superior mesenteric artery is a rare entity usually found in combination with aortic dissection. The isolated superior mesenteric artery dissection is even less frequent. In the current literature 168 cases are reported although according to Foord and Lewis the incidence found in autopsies is $0.06 \%$ [2]. Its clinical presentation varies from asymptomatic to life-threatening bowel ischemia or arterial rupture. Predisposing factors in the emergence of this dissection include hypertension, medial degeneration, atherosclerosis, fibromuscular dysplasia, segmental arterial mediolysis, connective tissue disorders, and trauma [8]. This is the first time an isolated dissection after ESWL is reported.
The exact mechanism of vessel injuries after ESWL is still undefined. However, the effects of ultrasound on blood vessels have been studied over the last years. Miller et al. suggested that micro bubbles constrained in blood vessels, after being excited by ultrasound, may damage the endothelium and rupture the vessel wall [3]. This is supported by Chen and his colleagues who showed that bubbles undergo oscillations which deform the vessels on the same scale [4]. The same author performed an experiment on ex vivo rat mesentery, suggesting that the vessel wall disruption after ultrasound is caused by vessel distention or invagination, due to cavitation bubbles [5]. The calcification in the aorta potentiates these effects by acting as an acoustic interface and leads to plaque rupture, especially in areas such as the transitional point from the fixed to mobile part of the SMA.

There are no evidence-based guidelines regarding the use of such therapeutic strategy. Options include the medical approach with or without anticoagulation, open surgery, and endovascular therapy. The decision of therapy was based upon the patient's response to initial treatment, that is, blood pressure control and anticoagulation treatment with unfractionated heparin. Park et al. reported a series of 58 patients with isolated superior mesenteric artery dissection, 53 of them being treated conservatively, 4 surgically, and 1 by SMA stenting [6]. The decision to intervene was based on signs of bowel ischemia and unremitting pain after one week of medical therapy. Most patients presented with acute abdominal pain which subsided after a few days and was attributed to the dissection solely. After 23 months of followup, $43 \%$ of patients showed no angiographic change of dissection, $41.3 \%$ showed a diminished extent of false lumen, and $15.2 \%$ showed a complete remodeling, with most changes occurring in the first 6 months.

Concerning the use of antithrombotic therapy, there is a discrepancy among articles. Several authors support the use of anticoagulation [9], while others consider anticoagulation to oppose the goal of treatment, which is to thrombose and obliterate the false lumen [10]. In this case, we used anticoagulation with heparin intravenously, with subsequent conversion to warfarin orally, as the patient's symptoms subsided the first hours after initial treatment.

In conclusion, the rarity of this case surely does not support a thorough vascular screening test in patients who will receive shockwave treatment. However, this complication should be kept in mind whenever a patient develops acute abdominal pain after this procedure. The treatment should be optimized according to the patient's clinical status and should be revised in case of symptom aggravation.

\section{Abbreviations}

SME: Superior mesenteric artery

ESWL: Extracorporeal shockwave lithotripsy

WBC: White blood cells CRP, C-reactive protein.

\section{Conflict of Interests}

C. Bakoyiannis and other coauthors declare that they have no conflict of interests. 


\section{References}

[1] E. Neri, G. Capannini, F. Diciolla et al., "Localized dissection and delayed rupture of the abdominal aorta after extracorporeal shock wave lithotripsy," Journal of Vascular Surgery, vol. 31, no. 5, pp. 1052-1055, 2000.

[2] A. G. Foord and R. D. Lewis, "Primary dissecting aneurysms of peripheral and pulmonary arteries: dissecting hemorrhage of media," Archives of Pathology \& Laboratory Medicine, vol. 68, pp. 553-771, 1959.

[3] D. L. Miller, M. A. Averkiou, A. A. Brayman et al., "Bioeffects considerations for diagnostic ultrasound contrast agents," Journal of Ultrasound in Medicine, vol. 27, no. 4, pp. 611-632, 2008.

[4] H. Chen, W. Kreider, A. A. Brayman, M. R. Bailey, and T. J. Matula, "Blood vessel deformations on microsecond time scales by ultrasonic cavitation," Physical Review Letters, vol. 106, no. 3, Article ID 034301, 2011.

[5] H. Chen, A. A. Brayman, M. R. Bailey, and T. J. Matula, "Blood vessel rupture by cavitation," Urological Research, vol. 38, no. 4, pp. 321-326, 2010.

[6] Y. J. Park, K. B. Park, D. I. Kim et al., "Natural history of spontaneous isolated superior mesenteric artery dissection derived from follow-up after conservative treatment," Journal of Vascular Surgery, vol. 54, no. 6, pp. 1727-1733, 2011.

[7] M. K. Lazarides, H. Drista, D. P. Arvanitis, and J. N. Dayantas, "Aortic aneurysm rupture after extracorporeal shock wave lithotripsy," Surgery, vol. 122, no. 1, pp. 112-113, 1997.

[8] M. N. Tameo, M. J. Dougherty, and K. D. Calligaro, "Spontaneous dissection with rupture of the superior mesenteric artery from segmental arterial mediolysis," Journal of Vascular Surgery, vol. 53, no. 4, pp. 1107-1112, 2011.

[9] T. Nagai, R. Torishima, A. Uchida et al., "Spontaneous dissection of the superior mesenteric artery in four cases treated with anticoagulation therapy," Internal Medicine, vol. 43, no. 6, pp. 473-478, 2004.

[10] S. I. Min, K. C. Yoon, S. K. Min et al., "Current strategy for the treatment of symptomatic spontaneous isolated dissection of superior mesenteric artery," Journal of Vascular Surgery, vol. 54, no. 2, pp. 461-466, 2011. 


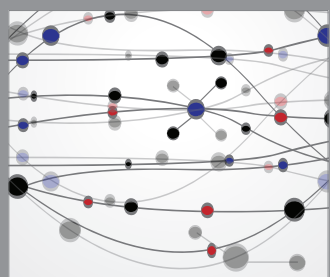

The Scientific World Journal
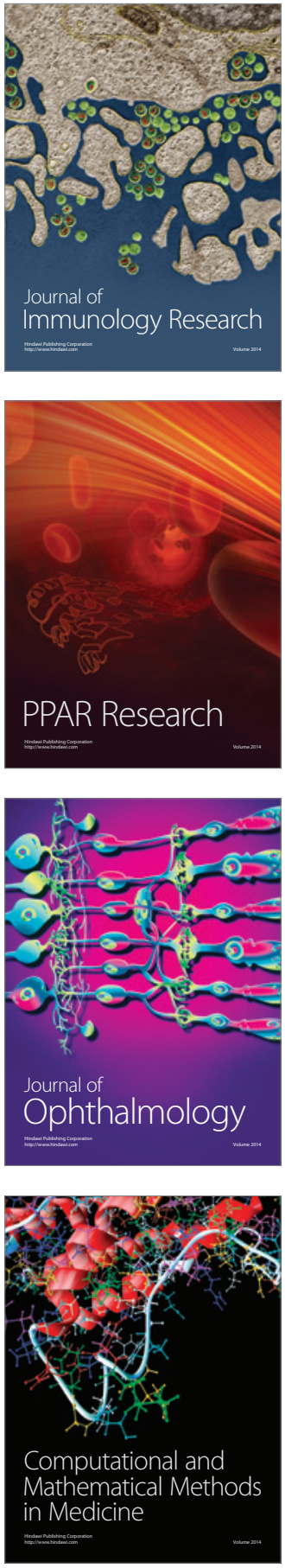

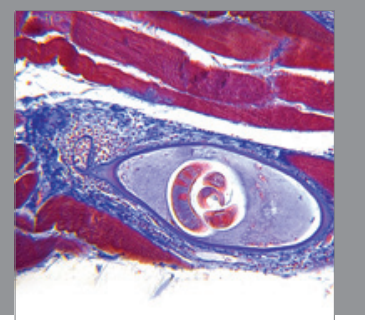

Gastroenterology

Research and Practice
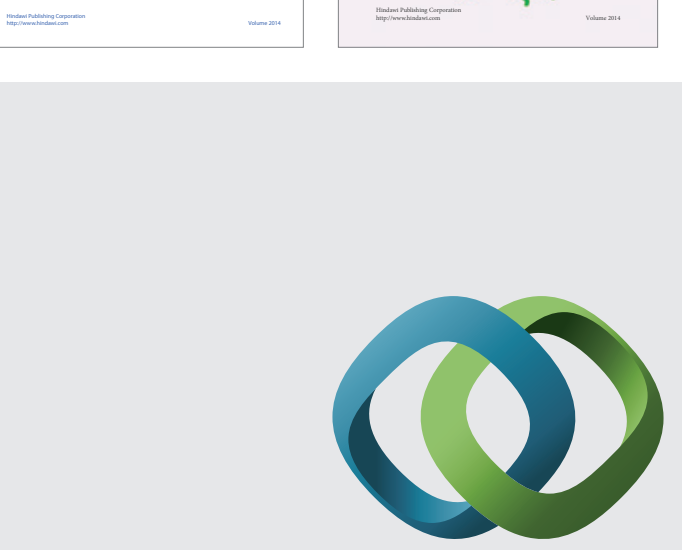

\section{Hindawi}

Submit your manuscripts at

http://www.hindawi.com
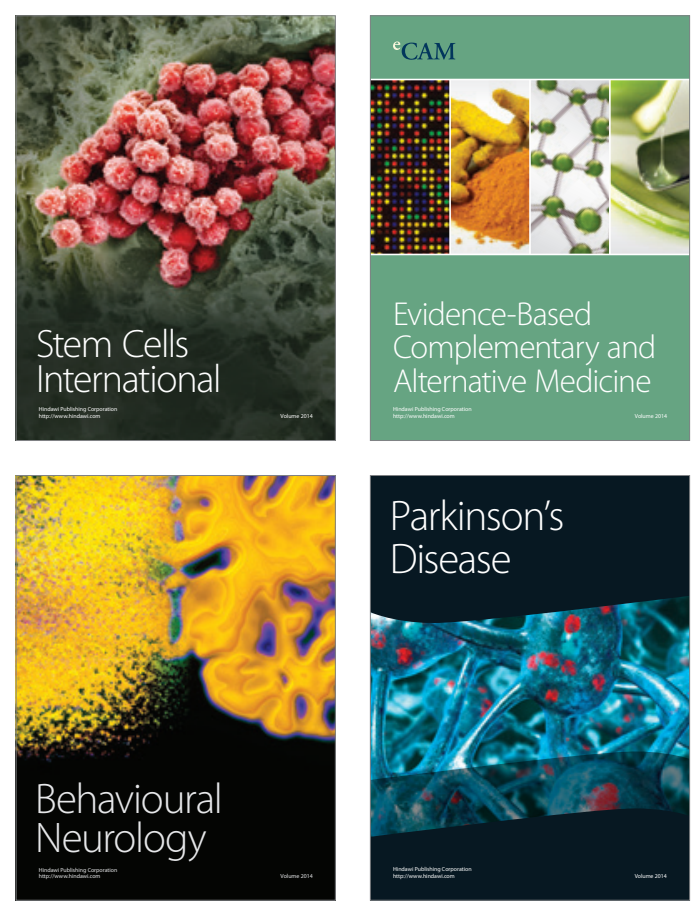

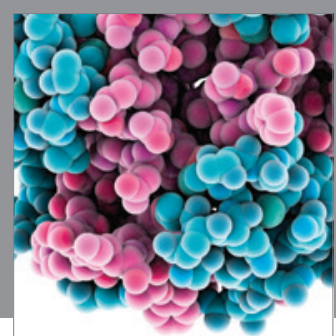

Journal of
Diabetes Research

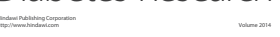

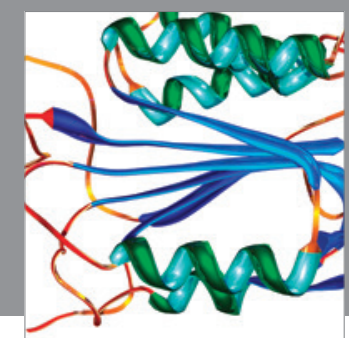

Disease Markers
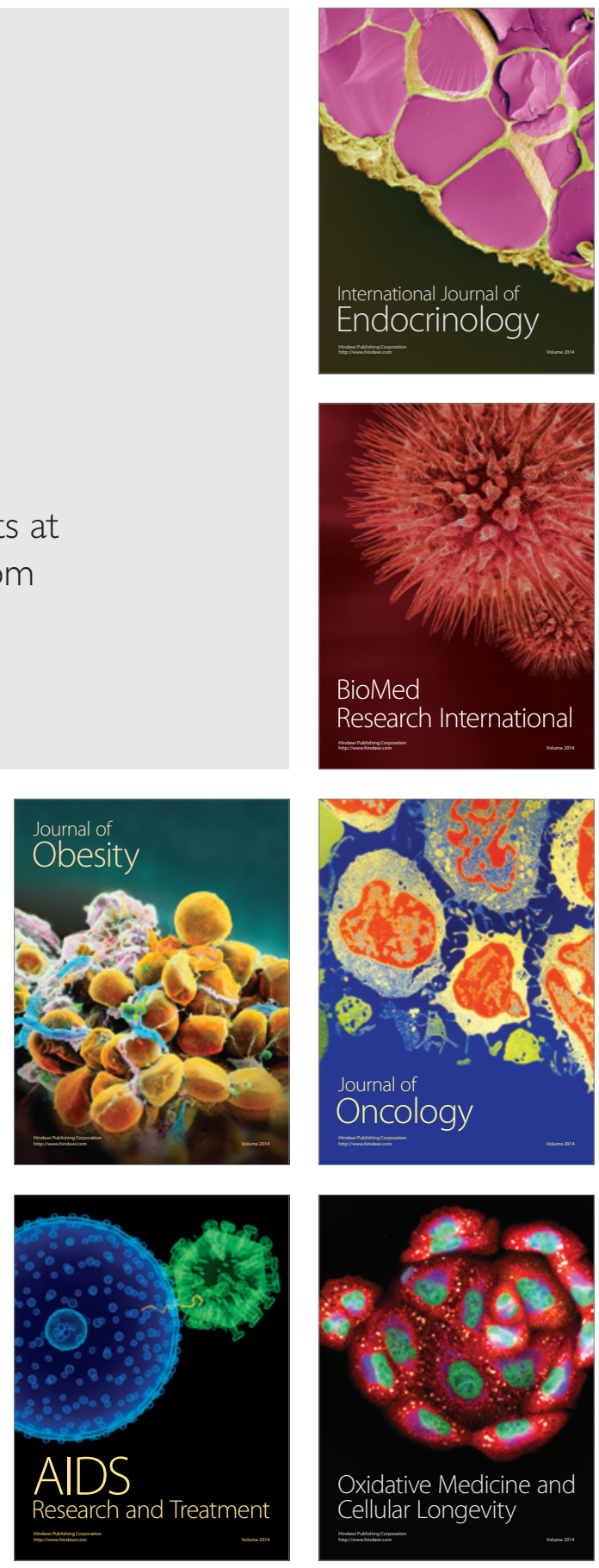